\title{
Approaches for reducing structural vibration of the carbody railway vehicles
}

\author{
Mădălina Dumitriu* and Cătălin Cruceanu \\ ${ }^{1}$ University Politehnica of Bucharest, Department of Railway Vehicles, 313 Splaiul Independenţei, \\ 060042, Bucharest, Romania
}

\begin{abstract}
Reducing the weight of the railway vehicles stands as a decisive rule in their design, entailed by higher velocities, the need to consume less energy and lower the manufacturing costs, along with the maximization of the use of loads on the axle. Once complied with this rule, the vehicle flexibility increases and leads to an easy excitation of the structural vibrations in the carbody, with an impact upon the ride comfort in the railway vehicle. For a better ride comfort in lightweight railway vehicles, both vibration isolation approaches and structural damping approaches have been introduced. The paper herein submits a brief review of the main structural damping approaches aiming to reduce the amplitude in the carbody structural vibrations, based on the use of the piezoelectric elements in passive control schemes. The paper outcomes show the potential of the presented methods concerning the reduction of the flexible vibrations in the carbody and the ride comfort improvement.
\end{abstract}

\section{Introduction}

A lower weight is a basic criterion in designing the railway vehicles with respect to reaching higher velocities with the lowest energy consumption possible. The weight decrease of the railway vehicles also contributes to the reduction of ground vibration and construction-cost saving [1]. Similarly, the demand from the transportation service providers to maximize usage of the limited axle loads compel the railway industry to adopt the lightweight carbody designs $[2,3]$. While the requirements of strength and crashworthiness are complied with, the reduction in the carbody weight is usually done by a significant decrease of the structural stiffness [2]. The lighter the vehicle carbody, the higher its flexibility, thus allowing an easier excitation of the carbody structural vibrations. The structural vibration leads to carbody fatigue which affects the dynamic performances and service life of the vehicle [4]. There are likewise numerous theoretical and experimental studies to validate that the level of carbody vibrations can be strongly influenced by the flexible modes of the carbody vibrations, mainly for the high speed vehicles [2, 4 - 6]. In some cases, this carbody structural flexibility accounts for half of the perceived vibrations, while the rest is due to rigid modes [7].

The value of the frequencies for the first structural modes of the carbody vibration often fall within the frequency range of high human sensitivity to vibration. As a result, structural

\footnotetext{
*Corresponding author: madalinadumitriu@yahoo.com
} 
vibrations of the vehicle carbody have a great impact on the ride comfort, both for high speed trains $[2,8]$ and for the commuter or metro trains $[1,3,9]$. In spite of the fact that the carbody structural vibrations are rather complex [7], the greatest influence upon the ride comfort comes from the first carbody natural bending mode $[6,10]$ whose frequency usually ranges from 6 to $12 \mathrm{~Hz}$, an interval where the human body shows a higher sensitivity to vertical vibrations.

The conventional solution to reduce the structural vibrations of the vehicle carbody involves an increase in the carbody stiffness. Different solutions can be adopted to come to this situation [11]. One is to have a carbody as short as possible but this solution contradicts the desire to maximise the number of passengers in relation to the number of bogies, as an example. Another one aims the carbody cross-section, which should be the largest possible. However, the cross-section must not interfere with the prescribed carbody gauge profile. Furthermore, crosswind stability problems may arise. Stiffer yet heavier carbodies can be designed but this is nonetheless in opposition to the lightweight design targets. Similarly, the necessary reinforcements for the carbody stiffening often lead to a more complicated and therefore more expensive manufacturing of the carbody [3].

More concepts on the reduction of the comfort-relevant vibrations of the carbody vibrations have already been introduced. They can be classified into two complementary approaches, namely the vibration isolation approaches to include passive, semi-active and active concepts with the role of stopping the excitation propagation through the suspension and structural damping approaches - passive or active - with the aim at reducing the carbody structure vibration amplitudes [9].

This paper will provide a brief review of the main carbody structural damping approaches, based on the use of the piezoelectric elements in passive control schemes. The piezoelectric elements are used in passive control schemes to suppress the flexible vibration modes of the carbody and, hence, to improve the ride comfort by benefiting from the ability of the piezoelectric elements to convert the mechanical energy into electrical energy.

\section{Passive damping structural approaches}

The concepts regarding the passive structure damping of the railway vehicle carbody rely on the ability of the piezoelectric elements to convert the mechanical energy, i.e. the vibration energy in the structure, into electrical energy.

Takigami [12] applies this concept in order to develop a new technique to suppress the bending vibration of railway vehicle carbodies that use piezoelectric elements which are electrically shunted by an external circuit (shunt circuit). When the vibration occurs as shown in Fig. 1, a strain is produced in the piezoelectric elements attached to the carbody which is deformed. As a result, a voltage is generated. When a shunt circuit including a resistor is connected to the piezoelectric elements, the voltage generated by the elements causes a current to flow to the circuit. As the current flows through the resistor in the shunt circuit, the electric energy is dissipated in the form of Joule heat, triggering a loss of energy. The energy loss has a damping effect on the carbody, thereby suppressing the bending vibration. The suggested theory is tested on a 1:5 scale model of Shinkansen carbody model, with eight piezoelectric elements - four on each of the lateral side beams - and to a stationary vibration test. The results of the vibrations test in Fig. 2 show that the acceleration amplitude at the carbody centre has lowered to less than half of its value.

The same method for improving the ride comfort through reducing the flexible vibrations in the railway vehicles carbody by means of the piezoelectric elements attached on the carbody is also submitted by Hansson [13]. 


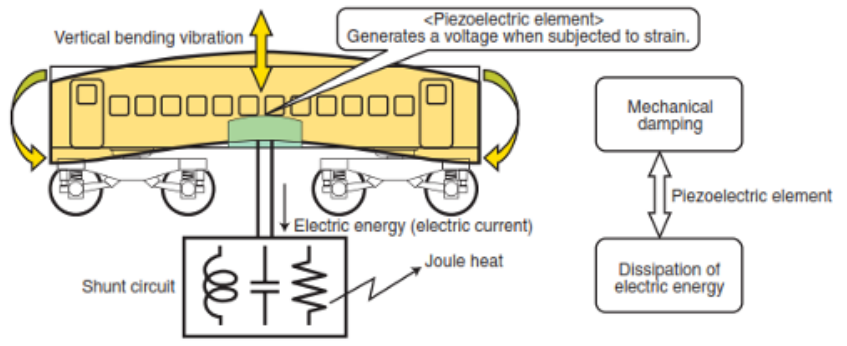

Fig. 1. Scheme of bending vibration suppression technique [12].

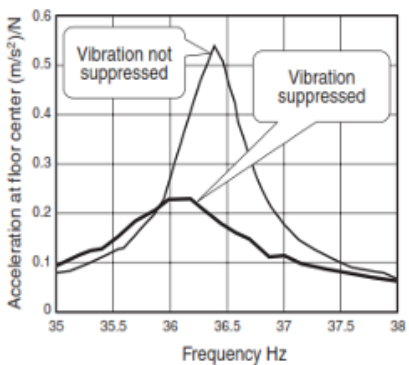

Fig. 2.Vibration test results [12].

The efficiency of the method is both theoretically and experimentally researched. Two types of electric shunt circuits are studied; a single-mode shunt circuit which is tuned for a single mode damper, and a multi-mode shunt circuit for two-mode damping. The piezoelectric material (lead zirconate titanate) (LZT) is bonded to a host structure and the electrodes of the piezoelectric element are shunted with some electrical impedance.

Should the shunt consist of an inductor and resistor in series, the shunt combined with the capacitance of the piezoelectric element creates a damped electrical resonance. The resonance can be tuned so that the piezoelectric device acts as a damped vibration absorber. A damped vibration absorber replaces a single structural mode with two highly damped modes. (Fig. 3, a). To simultaneously suppress multiple modes, one multi-resonant shunt circuit is used (Fig. 3, b).

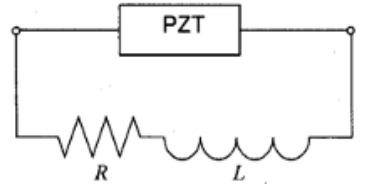

a. Single mode damping.

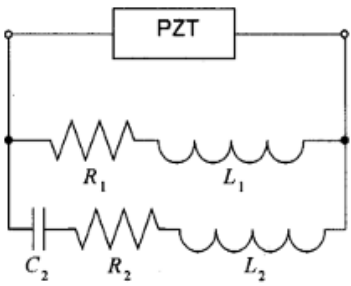

b. Two-mode damping.

Fig. 3. Shunt circuits [13].

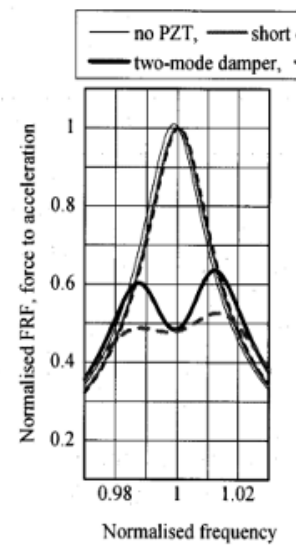

(a) $\omega / \omega_{1}$

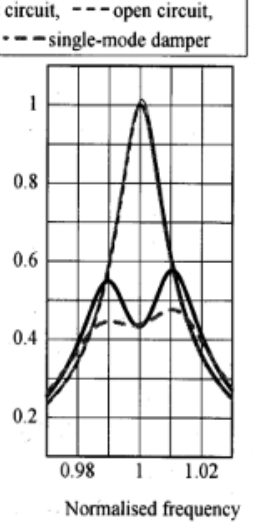

(b) $\omega / \omega_{3}$

Fig. 4. Simulated FRF peak reduction at the first (a) and third (b) bending modes, near the centre of the carbody [13].

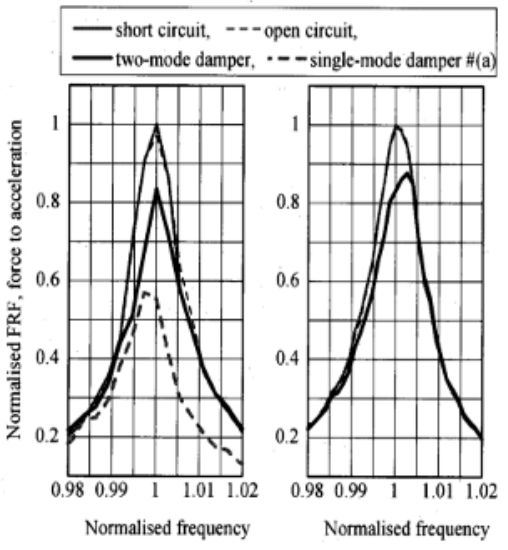

(a) $\omega / \omega_{1}$

(b) $\omega / \omega_{2}$

Fig. 5. Measured FRF peak reduction at the first bending mode (a) and at a local mode (b) at 76.2 $\mathrm{Hz}$ near the centre of the carbody [13]. 
To estimate the effectiveness of the piezoelectric damping, a 1:5 scale model of a suspended Shinkansen vehicle is investigated experimentally and numerically, both with and without piezoelectric elements. The results in Fig. 4 correspond to the numerical simulations. The frequency response function (FRF) value is shown to be reduced at the first bending mode peak by $48 \%$ for the single-mode damper and by $37 \%$ for the two-mode damper. The two-mode damper simultaneously reduces the frequency response value at the third bending mode peak by $43 \%$. In case the single-mode damper is targeted at the third bending mode instead of the first, the peak value can be reduced by 53\%. Figure 5 shows the measurement test results. When applying the single-mode damper, the FRF peak value at the first bending mode is reduced by $43 \%$. The two-mode damper reduces the FRF peak value at the first bending mode by $17 \%$ and the peak value at the local mode at $76.2 \mathrm{~Hz}$ by $12 \%$, at the same time.

The study shows that the effectiveness of the vibration suppression depends on the placement, size and amount of piezoelectric elements, where the placement should be as far away from the neutral axis of the car body as possible. The multi-mode damper is useful when the suppression of more vibration modes within the comfort-sensitive frequency interval is desired. Alternatively, the multi-mode damper cannot exceed the performance of the single-mode damper to suppress a single vibration mode.

Kamada et al. [14] proposed new control methods for shunt damping using stack type piezoelectric transducers (PZTs) and the effectiveness and the robustness of the designed circuits were confirmed through 1:6 scale model experiments that have almost the same eigenfrequencies of the first five modes as the Shinkarisen light carbody.

The railway vehicle is modelled as a simple beam supported on two points by a spring in parallel with a damper (see. Fig. 6). The stack type PZTs are attached at the centre of the beam. For the study, the first three bending modes in the total system were taken into account.

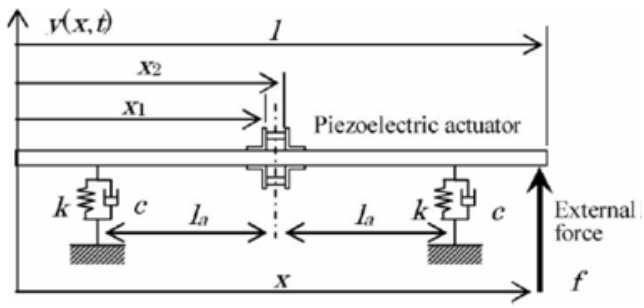

Fig. 6. Analytical model [14].

Two shunt circuits have been investigated, where one is the LR-LRC shunt and the other is the negative capacitance shunt. A single vibration mode is reduced to a simple LR circuit. The multiple damping is done by parallel connection of a RLC circuit to the original LR circuit. The LR-LRC circuit was designed to suppress the first and third bending modes of the beam.

The tests results are incorporated in Fig. 7 - 9. Fig. 7 shows a frequency response of LR shunt. On the left, a third vibration mode is efficiently suppressed since the circuit has been thus designed, which is not valid for the fifth vibration mode. Similarly, the LR shunt used on the right is only efficient for the fifth vibration mode. Fig. 8 includes the experimental results for the LR - LRC shunt. Both first and third bending modes are noted to have been effectively suppressed. The reduction rates are $29 \%$ for the third mode and $69 \%$ for the fifth one, which were a bit worse than the individual shunt damping by a single LR circuit. The results for the negative CR shunt are shown in Fig. 9, confirming that they are efficient for the suppression of both vibration modes and that the performance is better than for other circuits. 


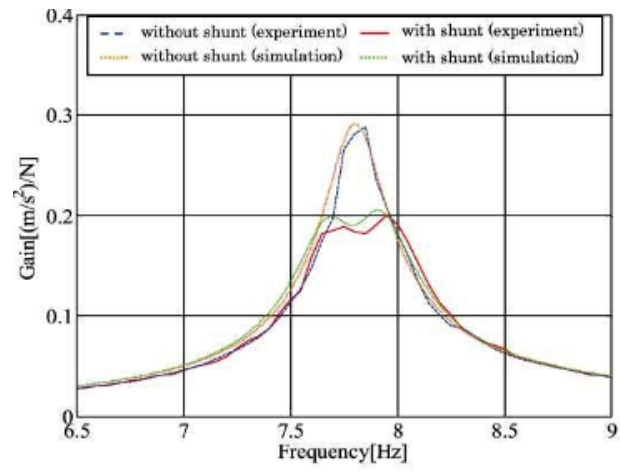

(a) Tuned for the 3 rd mode

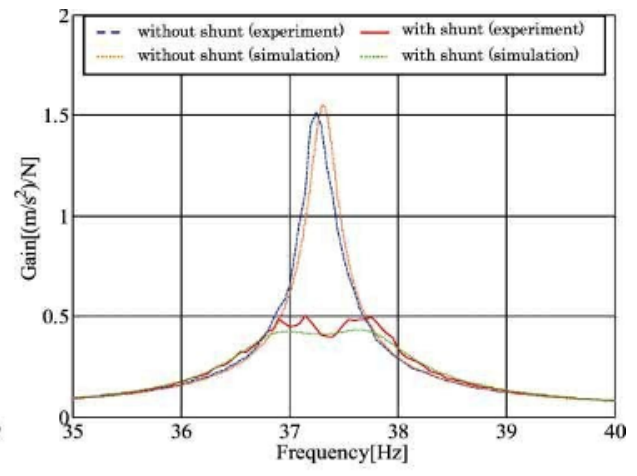

(b) Tuned for the 5 th mode

Fig. 7. Frequency responses of the beam acceleration by single LR shunt [14].
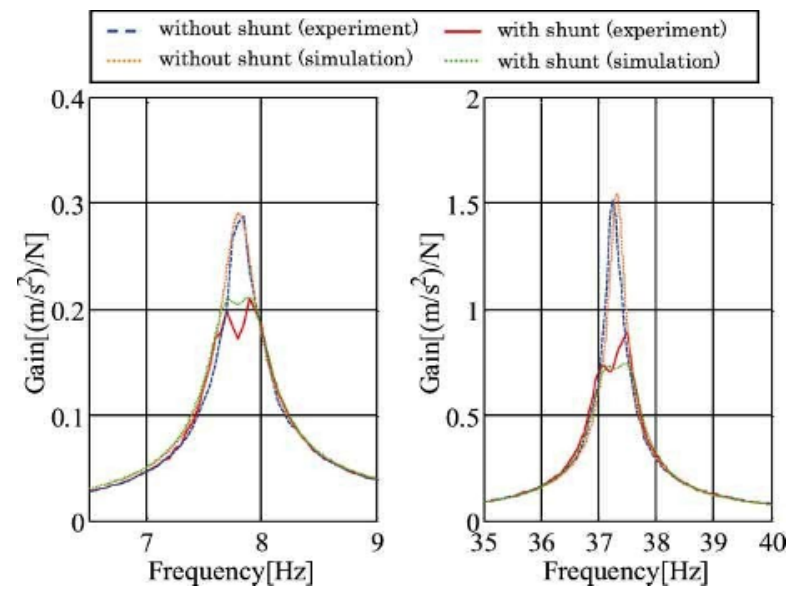

Fig. 8. Frequency responses by LR-LRC shunt [14].

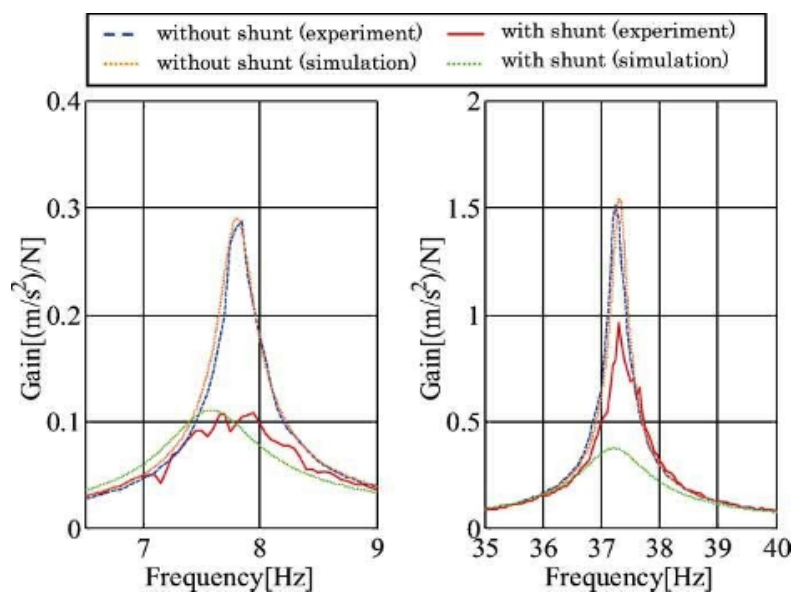

Fig. 9. Frequency responses by negative CR shunt [14].

Based on the results above, the conclusion is that both LR shunt and the negative capacitance shunt are efficient for the suppression of a single vibration mode of the carbody in the railway vehicles. The multiple vibration modes can be suppressed by parallel connection of a LR with a LRC circuit. 


\section{Conclusions}

The concern for lighter railway vehicles so as they are able to travel at higher speeds with less energy consumption and reduced manufacturing costs while having a maximum use of the loads on the axle has taken shape in a less heavy vehicle carbody. But the weight decrease is usually done by a significant decline in the structural stiffness that fosters the easy excitation of the flexible vibrations in the carbody. At this rate, the level in the carbody vibrations goes up and the ride comfort will be significantly affected if the increase occurs in the frequency range where the human body has a higher sensitivity to vibrations.

For the ride comfort, the literature of review introduces more concepts regarding the reduction in the carbody vibrations, which can be classified into vibration isolation approaches and structural damping approaches.

The paper herein submits the main structural damping approaches based on the use of the piezoelectric elements in the passive systems to control the carbody flexible vibrations. As a rule, the passive systems of controlling the carbody flexible vibrations rely on the ability of the piezoelectric elements attached to the carbody to convert the mechanical vibration energy into electrical energy that is later dissipated via a shunt circuit.

The studies described in this paper are a good evidence for the potential of the suggested methods concerning the reduction of the flexible vibrations in the carbody and the ride comfort improvement. The passive systems of the structural damping in the carbody herein featured can turn into an alternative to the active suspension that, in most cases, cannot be applied to improve the ride comfort due to the high costs of implementation and maintenance.

This work has been funded by University Politehnica of Bucharest, through the "Excellence Research Grants" Program, UPB - GEX - 2016. Research project title: Research on developing mechanical and numerical models for the virtual evaluation of the dynamic performances in the railway vehicles (in Romanian). Contract number: 48/26.09.2016.

\section{References}

1. T. Tomioka, T. Takigami, Y. Suzuki, Veh. Syst. Dyn 44, Suppl., 272-285 (2006)

2. J. Zhou, R. Goodall, L. Ren, H. Zhang, Proc. IMechE, Part F: J. of Rail and Rapid Transit 223, 461-471 (2009)

3. G. Schandl, P. Lugner, C. Benatzky et al. Veh. Syst. Dyn. 45, 9, 835-847 (2007)

4. G. Yang, C. Wang, F. Xiang, S. Xiao, Chinese J. Mech. Eng. 29, 6, 1120-1126 (2016)

5. M. Dumitriu, Archive of Mechanical Engineering LXI, 4, 609-625 (2014)

6. G. Diana, F. Cheli, A. Collina et al., Veh. Syst. Dyn 38, 3, 165-183 (2002)

7. P. Carlbom, Carbody and Passengers in Rail Vehicle Dynamics (Doctoral Thesis, Stockholm, 2000)

8. H. Shi, P. Wu, J. Mech. Sci. Technol. 30, 1, 55-66 (2016)

9. A. Schirrer, M. Kozek, Proceeding of the 6th Mediterranean Conference on Control and Automation Congress Centre, 481-486 (2008)

10. M. Dumitriu, J. Engineering Science and Technology Review 8, 4, 32-39 (2015)

11. A. Orvanäs, Methods for reducing vertical carbody vibrations of a rail vehicle (Report in Railway Technology Stockholm, 2010)

12. T. Takigami, Railway Technology Avalanche 9 (2005)

13. J. Hansson, M. Takano, T. Takigami, T. Tomioka, Y. Suzuki, JSME Int. J., Ser. C 47, 2 , 451-456 (2004).

14. T. Kamada, R. Kiuchi, M. Nagai, Veh. Syst. Dyn 46, Suppl., 561-570 (2008) 